\title{
SUR LE PROLONGEMENT DES FONCTIONS AFFINES
}

\author{
N. BOBOC et GH. BUCUR
}

0.

Soient $X$ un espace compact, $H$ un sous-espace de fonctions continues (réelles, complexes ou quaternioniques) qui contient les fonctions constantes et qui sépare les points de $X, F$ un sous-ensemble fermé de $X$ qui contient la frontière de Choquet de $X$ par rapport à $H$ et $H_{1}$ un sous-espace de fonctions continues (réelles, complexes ou quaternioniques) $H$-affines sur $X$. Le but de ce travail est de présenter des théorèmes de prolongement d'une fonction $H$-affine sur $F$ à une fonction de $H_{1}$. Nous étendons ainsi un résultat de E. Alfsen [1].

1.

Soient $X$ un espace compact, $\mathscr{C}(X, \mathrm{R})$ l'espace linéaire des fonctions réelles continues sur $X$ et $H$ un sous-espace linéaire de $\mathscr{C}(X, \mathrm{R})$ qui contient les fonctions constantes et qui sépare les points de $X$. Nous désignerons par $\mathscr{S}_{H}$ le cône convexe min-stable engendré par $H$, c'est-à-dire l'ensemble de fonctions continues $s$ sur $X$ pour lesquelles il existe une famille finie $\left(h_{i}\right)_{i \in I}$ d'éléments de $H$ telle que

$$
x \in X \Rightarrow s(x)=\inf _{i \in I} h_{i}(x) .
$$

Pour toutes deux mesures de Radon positives $\mu_{1}, \mu_{2}$, sur $X$ nous désignerons par

la relation

$$
\mu_{1} \ll_{H} \mu_{2}
$$

$$
s \in \mathscr{S}_{H} \Rightarrow \mu_{1}(s) \leqq \mu_{2}(s) .
$$

Cette relation est une relation d'ordre sur l'ensemble $\mathscr{M}_{+}(X)$ des mesures de Radon positives sur $X$. Si $x \in X$ et $\mu \in \mathscr{M}_{+}(X)$, la relation

est équivalente à la relation

$$
\mu \ll_{H} \varepsilon_{x}
$$

$$
h \in H \Rightarrow \mu(h)=h(x) .
$$

Reçu le 22 février 1966. 
Une mesure $\mu \in \mathscr{M}_{+}(X)$ s'appelle $H$-minimale (ou simple minimale) s'il n'existe pas des mesures $\nu \in \mathscr{M}_{+}(X), \nu \neq \mu$, telles que $\nu \ll_{H} \mu$.

On sait (voir par exemple [3, propositions 1.5 and 1.6]) que l'ensemble de mesures minimales est un sous-cône convexe de $\mathscr{M}_{+}(X)$ relativement complet et solide par rapport à l'ordre de $\mathscr{M}_{+}(X)$.

Une mesure de Radon réelle $\mu$ s'appelle $H$-minimale (ou simple minimale) si la mesure positive $|\mu|$ est minimale.

L'ensemble de points $x \in X$ pour lesquels la mesure $\varepsilon_{x}$ est minimale est appelé la frontière de Choquet de $X$ par rapport à $H$ et sera noté par $\partial_{H} X$.

Soit $F$ un sous-ensemble fermé de $X$. Une fonction $s$ sur $F$ (finie ou non) sera nommée $H$-concave si on ait

$$
x \in F, \mu \ll_{H} \varepsilon_{x}, \mu(X-F)=0 \Rightarrow \mu(s) \leqq s(x) .
$$

(Pour une mesure de Radon positive $\mu$ sur un espace compact $Y$ et pour une fonction $f$ sur $Y$ (finie ou non) nous noterons par $\mu(f)$ l'intégrale supérieure de $f$. Pour tout ensemble $A \subset X$ nous écrirons $\mu(A)$ au lieu de $\mu\left(\chi_{A}\right)$ où $\chi_{A}$ est la fonction caractéristique de $A$.) Une fonction $f$ sur $F$ (finie ou non) s'appelle $H$-affine si $f$ et $-f$ sont $H$-concaves sur $F$. Nous désignerons par $\mathscr{S}_{H}{ }^{*}$ le cône convexe de fonctions $H$-concaves sur $X$ qui sont inférieurement bornées et inférieurement semicontinues. Un ensemble $A \subset X$ est dit $H$-frontière si la relation suivante est satisfaite:

$$
s \in \mathscr{S}_{H}{ }^{*}, s \geqq 0 \text { sur } A \Rightarrow s \geqq 0 \text { sur } X .
$$

On sait (voir par exemple [3, corollary 2-1]) qu'un ensemble $A \subset X$ est $H$-frontière si et seulement si on ait $A \supset \partial_{H} X$, c'est-à-dire que la frontière de Choquet de $X$ par rapport à $H$ est le plus petit ensemble $H$-frontière.

Puisque (voir par exemple [4], [5]) toute fonction $f \in \mathscr{S}_{H}{ }^{*}$ est l'enveloppe supérieure d'une famille filtrante croissante de fonctions $f^{\prime} \in \mathscr{S}_{H}$, pour qu'un ensemble fermé $F \subset X$ soit $H$-frontière il faut et il suffit que

$$
h \in H, h \geqq 0 \text { sur } F \Rightarrow h \geqq 0 \text { sur } X .
$$

De même il en résulte que pour toutes deux mesures $\mu, v \in \mathscr{M}_{+}(X)$ telles que

$$
\mu \ll_{H} \nu, \quad \mu(X-F)=v(X-F)=0
$$

où $F$ est un ensemble fermé, et toute fonction $H$-concave $f$ sur $F$ inférieurement semicontinue et inférieurement bornée, nous avons $\mu(f) \leqq v(f)$.

Soit $F$ un ensemble fermé $H$-frontière et $f$ une fonction sur $F$ (finie ou non). Nous désignerons par $\bar{Q}_{f}{ }^{F}, Q_{f}{ }^{F}$ les fonctions sur $X$ definies par 


$$
\begin{aligned}
& \bar{Q}_{f}{ }^{F}=\inf \left\{r(x) \mid s \in \mathscr{S}_{H}{ }^{*}, s \geqq f \operatorname{sur} F\right\}, \\
& \underline{Q}_{f}{ }^{F}=\sup \left\{t(x) \mid-t \in \mathscr{S}_{H}{ }^{*}, t \leqq f \text { sur } F\right\} .
\end{aligned}
$$

(Voir H. Bauer [2].) On peut voir que la fonction $\bar{Q}_{f}{ }^{F}$ est $H$-concave, que

et que

$$
\underline{Q}_{f}^{F}=-\bar{Q}_{-f} F, \quad \underline{Q}_{f}^{F} \leqq \bar{Q}_{f}^{F},
$$

$$
x \in F \Rightarrow \underline{Q}_{f}{ }^{F}(x) \leqq f(x) \leqq \bar{Q}_{f}{ }^{F}(x) .
$$

D'autre part, si la fonction $f$ est supérieurement semicontinue et supérieurement bornée nous aurons

$$
\bar{Q}_{f}^{F}(x)=\inf \{h(x) \mid h \in H, h \geqq f \text { sur } F\}
$$

et la fonction $\bar{Q}_{f}{ }^{F}$ sera supérieurement semicontinue.

Nous rappelons le lemme suivant (voir [3, lemme 1-1]):

Lemme. Soient $F$ un ensemble fermé $H$-frontière et $f$ une fonction supérieurement semicontinue, supérieurement bornée sur $F$. Alors pour toute mesure $\mu \in \mathscr{M}_{+}(X)$ nous avons

$$
\mu\left(\bar{Q}_{f}{ }^{F}\right)=\sup \left\{v(f) \mid v \ll_{H} \mu, \nu(X-F)=0\right\}
$$

et il existe une mesure $\mu_{0}, \mu_{0} \ll_{H} \mu, \mu(X-F)=0$ telle que

$$
\mu\left(\bar{Q}_{f}{ }^{F}\right)=\mu_{0}(f) .
$$

La mesure $\mu_{0}$ peut être choisie $H$-minimale si la fonction - $f$ est $H$-concave sur $F$.

De ce lemme il résulte en particulier qu'un ensemble fermé $F$ est $H$-frontière si et seulement si pour tout $x \in X$ il existe $\mu \ll_{H} \varepsilon_{x}$ telle que $\mu(X-F)=0$. De même pour tout ensemble fermé $H$-frontière $F$, le support de toute mesure $H$-minimale est contenue dans $F$.

2.

Soit $F$ un ensemble fermé $H$-frontière et $f$ une fonction réelle continue, affine sur $F$. Dans la suite nous donnerons une condition nécessaire et suffisante pour que $f$ peut être prolongée à une fonction réelle affine, continue sur $X$, ou peut être prolongée à une fonction de $H_{1}$, où $H_{1}$ est un sous-espace fermé de $\mathscr{C}(X, \mathrm{R})$ de fonctions affines sur $X$. Nous obtenons ainsi un résultat qui généralise un théorème de $\mathrm{E}$. Alfsen [1]. 
Proposition 1. Soient $F$ un ensemble fermé $H$-frontière et $f$ une fonction réelle continue sur $F$. Alors $f$ est $H$-affine sur $F$ si et seulement si

$$
x \in F \Rightarrow \bar{Q}_{f}^{F}(x)=\underline{Q}_{f}^{F}(x) .
$$

DÉmonstration. Si nous avons

alors

$$
\bar{Q}_{f}^{F}=\underline{Q}_{f}{ }^{F} \text { sur } F,
$$

$$
f=\bar{Q}_{f}{ }^{F}=\underline{Q}_{f}{ }^{F} \text { sur } F,
$$

et il résulte que $f$ est $H$-affine à cause du fait que $\bar{Q}_{f}{ }^{F}, \bar{Q}_{-f}{ }^{F}$ sont $H$-concaves.

Supposons que $f$ est $H$-affine sur $F$. Alors pour tout $x \in F$ il existe une mesure $\mu_{x}$,

$$
\mu_{x} \ll_{H} \varepsilon_{x}, \quad \mu_{x}(X-F)=0
$$

telle que $\bar{Q}_{f}^{F}(x)=\mu_{x}(f)$. Donc

$$
\bar{Q}_{f} F(x)=\mu_{x}(f)=f(x) .
$$

Puisque $-f$ est aussi $H$-affine sur $F$, nous avons

$$
x \in F \Rightarrow \bar{Q}_{-f} F(x)=-f(x) .
$$

Donc $\bar{Q}_{f} F(x)=\underline{Q}_{f} F(x)=f(x)$ pour tout $x \in F$.

La proposition 1 a été obtenue aussi par H. Bauer [2, Satz 4].

THÉORÈme 1. Soient $F$ un ensemble fermé $H$-frontière et $f$ une fonction réelle, continue, $H$-affine sur $F$. Alors il existe une fonction réelle, continue, $H$-affine $\tilde{f}$ sur $X$ telle que $\tilde{f}_{\mid F}=f$ si et seulement si pour tout $x \in X$ et toutes deux mesures $H$-minimales $\mu, v \in \mathscr{M}_{+}(X)$ telles que

on ait

$$
\mu, v \ll_{H} \varepsilon_{x}
$$

$$
\mu(f)=\nu(f) \text {. }
$$

Démonstration. La condition est évidemment nécéssaire. Supposons que $f$ satisfait à la condition annoncée. Nous montrerons que $\bar{Q}_{f}{ }^{F}=$ $\underline{Q}_{f} F$ d'où il résulte que la fonction réelle

$$
\tilde{f}=\bar{Q}_{f}{ }^{F}=\underline{Q}_{f}{ }^{F}
$$

est continue, $H$-affine sur $X$ et égale à $f$ sur $F$.

Soit $x \in X$. Du lemme il existe deux mesures $H$-minimales $\mu^{\prime}, \mu^{\prime \prime}$,

$$
\mu^{\prime} \ll_{H} \varepsilon_{x}, \quad \mu^{\prime \prime} \ll_{H} \varepsilon_{x}
$$


telles que

$$
\bar{Q}_{f}^{F}(x)=\mu^{\prime}(f), \quad \underline{Q}_{f}{ }^{F}(x)=\mu^{\prime \prime}(f) .
$$

Par hypothèse, nous avons $\mu^{\prime}(f)=\mu^{\prime \prime}(f)$. Donc $\bar{Q}_{f}^{F}(x)=\underline{Q}_{f}^{F}(x)$.

ThÉoRÈme 2. Soient $F$ un ensemble fermé $H$-frontière, $f$ une fonction réelle continue, $H$-affine sur $F$ et $H_{1}$ un sous-espace fermé de $\mathscr{C}(X, \mathrm{R})$ de fonctions $H$-affines. Alors $f$ peut être prolongée à une fonction de $H_{1}$ si et seulement si pour toute mesure (réelle) $H$-minimale $\mu$ la relation

implique la relation

$$
h \in H_{1} \Rightarrow \mu(h)=0
$$

$$
\mu(f)=0 \text {. }
$$

Démonstration. La condition est évidemment nécessaire.

Soit $\mu$ une mesure (réelle) sur $F$ telle que

$$
h \in H \Rightarrow \mu(h)=0 \text {. }
$$

Pour achever la démonstration il suffit de démontrer que $\mu(f)=0$. Soit $\mu=\mu_{+}-\mu_{-}$. Du lemme il existe deux mesures $H$-minimales $\mu_{1}, \mu_{2}$ telles que

et telles que

$$
\mu_{1} \ll_{H} \mu_{+}, \mu_{2} \ll_{H} \mu_{-}
$$

Puisque

$$
\mu_{+}\left(\bar{Q}_{f}^{F}\right)=\mu_{1}(f), \quad \mu_{-}\left(\bar{Q}_{f}^{F}\right)=\mu_{2}(f) .
$$

$$
h \in H_{1} \Rightarrow \mu_{1}(h)=\mu_{+}(h)=\mu_{-}(h)=\mu_{2}(h),
$$

il résulte que $\mu_{1}(f)=\mu_{2}(f)$. En vertu de la proposition 1 nous avons

Donc $\mu_{+}(f)=\mu_{-}(f)$.

$$
\bar{Q}_{f}^{F}=\underline{Q}_{f}^{F}=f \quad \text { sur } F .
$$

3.

Soient $\left(X_{i}\right)_{i \in I}$ une famille finie d'espaces compacts et $X=\oplus_{i \in I} X_{i}$ la somme topologique de $\left(X_{i}\right)_{i \in I}$. Considérons un sous-espace linéaire $H$ de $\mathscr{C}(X, \mathrm{R})$ qui sépare les points de $X$ et qui contient toute fonction $f$ sur $X$ telle que pour tout $i \in I$ la restriction $f_{\mid X_{i}}$ est constante. Pour tout $i \in I$ nous désignerons par $H_{i}$ le sous-espace linéaire de $\mathscr{C}\left(X_{i}, \mathrm{R}\right)$ définie par

$$
f \in H_{i} \Leftrightarrow \exists h \in H, \quad h_{\mid X_{i}}=f .
$$

On peut remarquer que pour toute mesure $\mu \in \mathscr{M}_{+}(X)$, tout $i \in I$ tels 
que $\mu\left(X_{i}\right)=\mu(X)$ et toute mesure $v \in \mathscr{M}_{+}(X)$ la relation $\nu \ll_{H} \mu$ est équivalente au couple de relations

$$
v\left(X_{i}\right)=v(X), \quad \nu \ll_{H_{i}} \mu .
$$

Il en résulte que pour tout ensemble fermé $F \subset X$, une fonction $f$ sur $F$ est $H$-concave (resp. $H$-affine) si et seulement si pour tout $i \in I$ la fonction $f_{\mid F \cap X_{i}}$ est $H_{i}$-concave (resp. $H_{i}$-affine).

De même, un ensemble $A \subset X$ est $H$-frontière si et seulement si, pour tout $i \in I, A \cap X_{i}$ est $H_{i}$-frontière. Donc on a

$$
\partial_{H} X=\oplus_{i \in I} \partial_{H_{i}} X_{i}
$$

Puisque chaque fonction de $\mathscr{S}_{H}{ }^{*}$ (c'est-à-dire une fonction $H$-concave, inférieurement semicontinue, inférieurement bornée) est l'enveloppe supérieure d'une famille filtrante croissante d'éléments de $\mathscr{S}_{H}$, nous avons que la relation $\mu \ll_{H^{\nu}}$ implique la relation

$$
s \in \mathscr{S}_{H}{ }^{*} \Rightarrow \mu(s) \leqq \nu(s) \text {. }
$$

Donc la relation $\mu \ll_{H} v$ est équivalente à la relation

$$
i \in I \Rightarrow \mu_{i} \ll_{H_{i}} \nu_{i}
$$

où pour tout $i \in I, \mu_{i}$ signifie la restriction de $\mu$ à $X_{i}$. De ce fait on déduit qu'une mesure $\mu \in \mathscr{M}_{+}(X)$ est $H$-minimale si et seulement si pour tout $i \in I$ la mesure $\mu_{i}=\mu_{\mid X_{i}}$ est $H_{i}$-minimale.

Soient $X$ un espace compact, C le corp complexe, $\mathscr{C}(X, \mathrm{C})$ l'espace linéaire des fonctions complexes continues sur $X$ et $H$ un sous-espace de $\mathscr{C}(X, \mathrm{C})$ qui contient les constantes et qui sépare les points de $X$.

Soient $X_{1}=X_{2}=X$ et $\mathscr{C}\left(X_{1} \oplus X_{2}, R\right)$ l'espace des fonctions réelles continues définies sur la somme topologique $X_{1} \oplus X_{2}$. Un élément $f$ de $\mathscr{C}\left(X_{1} \oplus X_{2}, \mathrm{R}\right)$ sera noté par $f_{1} \oplus f_{2}$ où $f_{1}$ (resp. $f_{2}$ ) est la restriction de $f$ à $X_{1}\left(\operatorname{resp} . X_{2}\right)$.

Une mesure réelle $\mu$ sur $X_{1} \oplus X_{2}$ sera notée par $\mu_{1} \oplus \mu_{2}$ où $\mu_{i}$ est la restriction de $\mu$ à $X_{i}$. Nous noterons par $\psi$ l'application linéaire réelle

$$
\psi: \mathscr{C}(X, \mathrm{C}) \rightarrow \mathscr{C}\left(X_{1} \oplus X_{2}, \mathrm{R}\right)
$$

définie de la manière suivante:

$$
f=f_{1}+i f_{2} \rightarrow \psi(f)=f_{1} \oplus f_{2},
$$

et par $\psi^{\prime}$ l'application de l'espace linéaire réel des mesures complexes sur $X$ dans l'espace de mesures réelles sur $X_{1} \oplus X_{2}$ définie de la manière suivante:

$$
\mu=\mu_{1}+i \mu_{2} \rightarrow \psi^{\prime}(\mu)=\mu_{1} \oplus\left(-\mu_{2}\right) \text {. }
$$


L'ensemble $\psi(H)$ est un sous-espace réel de $\mathscr{C}\left(X_{1} \oplus X_{2}, \mathrm{R}\right)$ tel que

$$
f_{1} \oplus f_{2} \in \psi(H) \Rightarrow\left(-f_{2}\right) \oplus f_{1} \in \psi(H) .
$$

Une fonction $f \in \mathscr{C}(F, C)$ où $F$ est un sous-ensemble fermé de $X$ s'appelle $H$-affine si la fonction $\psi(f)$ est $\psi(H)$-affine sur la somme topologique $F \oplus F$. On voit tout de suite que $f=f_{1}+i f_{2}$ est $H$-affine sur $F$ si et seulement si $f_{1}, f_{2}$ sont $(\operatorname{Re} H)$-affines sur $F$.

Une mesure complexe $\mu$ s'appelle $H$-minimale si la mesure $\psi^{\prime}(\mu)$ est $\psi(H)$-minimale. Evidemment $\mu=\mu_{1}+i \mu_{2}$ est $H$-minimale si et seulement si les mesures $\mu_{1}, \mu_{2}$ sont $(\operatorname{Re} H)$-minimales.

L'ensemble des points $x \in X$ tels que $\varepsilon_{x}$ est $H$-minimale s'appelle la frontière de Choquet de $X$ par rapport à $H$. Un ensemble $A \subset X$ est dit $H$-frontière si la somme topologique $A \oplus A$ est $\psi(H)$-frontière. On voit qu'un ensemble $F \subset X$ fermé est $H$-frontière si et seulement si pour tout $h \in H$ on a

$$
\sup _{x \in X}|h(x)|=\sup _{x \in \boldsymbol{F}}|h(x)| .
$$

De la définition d'une mesure $H$-minimale il en résulte que son support est contenu dans tout ensemble fermé $H$-frontière.

Soit $H_{1}$ un espace linéaire complexe de fonctions continues $H$-affines sur $X$. Puisque

$$
f_{1} \oplus f_{2} \in \psi\left(H_{1}\right) \Rightarrow\left(-f_{2}\right) \oplus f_{1} \in \psi\left(H_{1}\right),
$$

une mesure complexe $\mu$ s'annule sur $H_{1}$ si et seulement si la mesure réelle $\psi^{\prime}(\mu)$ s'annule sur $\psi\left(H_{1}\right)$.

ThÉoRÈme 3. Soient $F$ un ensemble fermé $H$-frontière, $f$ une fonction $H$-affine sur $F$ et $H_{1}$ un sous-espace complexe fermé de $\mathscr{C}(X, C)$ de fonctions $H$-affines sur $X$. Alors $f$ peut être prolonger à une fonction de $H_{1}$ si et seulement si pour toute mesure complexe $\mu, H$-minimale, la relation

implique la relation

$$
h \in H_{1} \Rightarrow \mu(h)=0
$$

$$
\mu(f)=0 .
$$

Démonstration. L'assertion résulte des considérations précédentes et du théorème 2.

Soient $X$ un espace compact, $\mathrm{K}$ le corp des quaternions, $\mathscr{C}(X, \mathrm{~K})$ l'espace linéaire sur $K$ des fonctions continues sur $X$ à valeurs dans $K$ et $H$ un sous-espace de $\mathscr{C}(X, \mathrm{~K})$ que contient les fonctions constantes et qui sépare les points de $X$

Soient $X_{0}=X_{1}=X_{2}=X_{3}=X$ et $\mathscr{C}\left(\oplus_{e=0}^{3} X_{e}, \mathrm{R}\right)$ l'espace des fonctions 
réelles continues définies sur la somme topologique $\bigoplus_{e=0}^{3} X_{e}$. Un élément $f$ de $\mathscr{C}\left(\oplus_{e=0}^{3} X_{e}, \mathrm{R}\right)$ sera noté par $\oplus_{e=0}^{3} f_{e}$ où $f_{e}$ est la restriction de $f$ à $X_{e}$. Nous désignerons par $\psi$ l'application linéaire réelle

$$
\psi: \mathscr{C}(X, \mathrm{~K}) \rightarrow \mathscr{C}\left(\oplus_{e=0}^{3} X_{e}, \mathrm{R}\right)
$$

définie de la manière suivante:

$$
f=f_{0}+i f_{1}+j f_{2}+k f_{3} \rightarrow \psi(f)=\oplus_{e=0}^{3} f_{e},
$$

et par $\psi^{\prime}$ l'application de l'espace linéaire réel des mesures quaternioniques sur $X$ dans l'espace des mesures réelles sur $\oplus_{e=0}^{3} X_{e}$ définie de la manière suivante:

$$
\mu=\mu_{0}+i \mu_{1}+j \mu_{2}+k \mu_{3} \rightarrow \psi^{\prime}(\mu)=\left(-\mu_{0}\right) \oplus \mu_{1} \oplus \mu_{2} \oplus \mu_{3} .
$$

Une fonction $f \in \mathscr{C}(F, \mathrm{~K})$ où $F$ est un ensemble fermé de $X$ s'appelle $H$-affine si la fonction $\psi(f)$ est $\psi(H)$-affine sur la somme topologique $F \oplus F \oplus F \oplus F$. Evidemment $f \in \mathscr{C}(F, \mathrm{~K})$ est $H$-affine sur $F$ si et seulement si pour toute mesure $\mu \in \mathscr{M}_{+}(F)$ et tout $x \in F$ la relation

$$
h \in H \Rightarrow \mu(h)=h(x)
$$

implique la relation $\mu(f)=f(x)$.

Une mesure quaternionique $\mu$ sur $X$ s'appelle $H$-minimale si la mesure $\psi^{\prime}(\mu)$ est $\psi(H)$-minimale. Si $x \in X$, la mesure $\varepsilon_{x}$ est $H$-minimale si et seulement si pour tout mesure $\mu \in \mathscr{M}_{+}(X)$ la relation

$$
h \in H \Rightarrow \mu(h)=h(x)
$$

implique $\mu=\varepsilon_{x}$. L'ensemble des points $x \in X$ tels que la mesure $\varepsilon_{x}$ est $H$-minimale s'appelle la frontière de Choquet de $X$ par rapport à $H$.

Un ensemble $A \subset X$ est dit $H$-frontière si la somme topologique $A \oplus A \oplus A \oplus A$ est $\psi(H)$-frontière. De la définition d'une mesure $H$-minimale il en résulte que son support est contenu dans tout ensemble fermé $H$-frontière.

Soit $H_{1}$ un sous-espace fermé de $\mathscr{C}(X, \mathrm{~K})$ de fonctions $H$-affines sur $X$. Puisque

$$
f_{0} \oplus f_{1} \oplus f_{2} \oplus f_{3} \in \psi\left(H_{1}\right) \Rightarrow\left\{\begin{array}{l}
f_{1} \oplus\left(-f_{0}\right) \oplus f_{3} \oplus\left(-f_{2}\right) \in \psi\left(H_{1}\right), \\
f_{2} \oplus\left(-f_{3}\right) \oplus\left(-f_{0}\right) \oplus f_{1} \in \psi\left(H_{1}\right), \\
f_{3} \oplus f_{2} \oplus\left(-f_{1}\right) \oplus\left(-f_{0}\right) \in \psi\left(H_{1}\right),
\end{array}\right.
$$

on déduit qu'une mesure quaternionique $\mu$ sur $X$ s'annulle sur $H_{1}$ si et seulement si la mesure réelle $\psi^{\prime}(\mu)$ s'annule sur $\psi\left(H_{1}\right)$.

THÉOR亡̀me 4. Soient $F$ un ensemble fermé $H$-frontière, $f$ une fonction $H$-affine sur $F$ et $H_{1}$ un sous-espace fermé de $\mathscr{C}(X, \mathrm{~K})$ de fonctions $H$-affines 
sur $X$. Alors $f$ peut être prolongèe à une fonction de $H_{1}$ si et seulement si pour toute mesure quaternionique $\mu, H$-minimale, la relation

implique la relation

$$
h \in H_{1} \Rightarrow \mu(h)=0
$$

$$
\mu(f)=0 \text {. }
$$

L'assertion résulte des considérations précédentes et du théorème 2.

Remarque. Les théorèmes 2 et 3 généralisent les résultats de E. Alfsen [1] puisque pour chaque mesure minimale, la complémentaire de la frontière de Choquet a la mesure intérieure (Baire) égale à zero (voir par exemple [2, Satz 2 und Satz 3]).

\section{BIBLIOGRAPHIE}

1. E. Alfsen, On the Dirichlet problem of the Choquet boundary, Acta Math. 120 (1968), 149-159.

2. H. Bauer, Silovscher Rand und Dirichletsches Problem, Ann. Inst. Fourier (Grenoble) 11 (1961), 89-136.

3. N. Boboc et A. Cornea, Convex cones of lower semicontinuous functions on compact spaces, Rev. Roumaine Math. Pures Appl. 12 (1967), 471-525.

4. D. A. Edwards, Minimum stable wedges of semi-continuous functions, Math. Scand. 19 (1966), 15-26.

5. G. Mokobodski, Quelques propriétés des fonctions numériques convexes sur un ensemble convexe compact, Seminaire Brelot-Choquet-Deny de la theorie du potentiel, $6^{e}$ Année (1961/62), Exposé no. 9, pp. 3.

\section{INSTITUT DE MATHEMATIQUE}

ACADEMIE DE LA REPUBLIQUE SOCIALISTE DE ROUMANIE, BUCAREST 